\title{
Genes Positively Selected in Domesticated Mammals Are Significantly Dysregulated in the Blood of Individuals with Autism Spectrum Disorders
}

\author{
Antonio Benítez-Burraco \\ Department of Spanish, Linguistics, and Theory of Literature (Linguistics), Faculty of Philology, University of Seville, \\ Seville, Spain
}

\section{Keywords}

Autism spectrum disorders - Differentially expressed genes · Domestication - Etiopathogenesis · Human evolution

\begin{abstract}
Human self-domestication (i.e., the presence of traits in our species that are commonly found in domesticated animals) has been hypothesized to have contributed to the emergence of many human-specific features, including aspects of our cognition and behavior. Signs of self-domestication have been claimed to be attenuated in individuals with autism spectrum disorders (ASD), this conceivably accounting for facets of their distinctive cognitive and behavioral profile, although this possibility needs to be properly tested. In this study, we have found that candidate genes for mammal domestication, but not for neural crest development and function, are significantly dysregulated in the blood of subjects with ASD. The set of differentially expressed genes (DEGs) is enriched in biological and molecular processes, as well as in pathological phenotypes, of relevance for the etiology of ASD, like lipid metabolism, cell apoptosis, the activity of the insulin-like growth factor, gene expression regulation, skin/ hair anomalies, musculoskeletal abnormalities, and hearing impairment. Moreover, among the DEGs, there are known
\end{abstract}

candidates for ASD and/or genes involved in biological processes known to be affected in ASD. Our findings give support to the view that one important aspect of the etiopathogenesis of ASD is the abnormal manifestation of features of human self-domestication.

(c) 2019 S. Karger AG, Basel

Autism spectrum disorders (ASD) are pervasive neurodevelopmental clinical conditions mostly impacting on social communication abilities and behavior [American Psychiatric Association, 2013]. Many genes have been associated with ASD, and several environmental factors have been hypothesized to contribute to ASD as well [Geschwind and State, 2015; Gyawali and Patra, 2019; among many others]. Nonetheless, the etiology of ASD is still unclear, particularly in absence of robust genotype-tophenotype correlations (see the SFARI Gene database [https://gene.sfari.org/] for the most updated account). It is also uncertain why ASD show such a high prevalence in humans and why this prevalence has seemingly remained stable in time [Baxter et al., 2015]. At the same time, it is expected that the multiple factors associated with ASD risk converge onto common molecular pathways and regulatory networks [Kumar et al., 2019; Sullivan et al., 2019].

\section{KARGER}

(c) 2019 S. Karger AG, Basel

E-Mail karger@karger.com

www.karger.com/msy
Antonio Benítez-Burraco

Department of Spanish, Linguistics, and Theory of Literature (Linguistics)

Faculty of Philology, University of Seville

C/ Palos de la Frontera s/n, ES-41004 Seville (Spain)

E-Mail abenitez8@us.es 
Because of the deep link that exists between evolution and abnormal development, with recently evolved neural networks (and recently acquired cognitive and behavioral abilities) being more sensitive to damage because of their reduced resilience [Toro et al., 2010], one way of disentangling the etiology of ASD might be focusing on the genes that are involved in recent evolutionary changes in our species. One of these changes is the development of many traits commonly found in domesticated species of animals, including morphological, physiological, and behavioral features [for review, see Hare, 2017]. According to the human self-domestication hypothesis, our species suffered a process of domestication in response to external factors (mostly social factors, like choosing less reactive partners for mating, living in community, or coparenting) that triggered, or contributed to, the emergence of central aspects of our behavioral phenotype, including our enhanced sociability and cooperation and our distinctive mode of communication [Wilkins et al., 2014; Hare, 2017; Thomas and Kirby, 2018]. Hence, and considering that, as noted, ASD involve behavioral, social, and communication problems, an intriguing possibility is that one etiological component of the ASD phenotype is the abnormal manifestation of human self-domestication features. This possibility is even more intriguing if one considers that the different traits associated with domestication have been hypothesized to result from one common underlying mechanism, namely, the hypofunction of the neural crest (NC), an embryonic structure giving rise to many body parts during development [Wilkins et al., 2014]. Accordingly, it could be further hypothesized that one core aspect of the etiopathogenesis of ASD might be NC hyperfunction.

Notably, altered self-domestication features have been found in other human-specific cognitive disorders, like schizophrenia [Benítez-Burraco et al., 2017] and Williams syndrome [Niego and Benítez-Burraco, 2019]. We have recently shown that the same is true for ASD, as subjects with these conditions exhibit attenuated characteristics of domestication [Benítez-Burraco et al., 2016]. Nonetheless, the possibility that an abnormal manifestation of human self-domestication features, impacting on cognition and behavior and resulting from NC dysfunction, contributes to the ASD phenotype needs to be properly tested. On the one hand, we need to conduct clinical surveys aimed to check whether the links found in the literature between domestication and ASD are statistically significant. On the other hand, we need to examine this overlapping between domestication and ASD at a molecular level, checking, in particular, whether domestica- tion and ASD share some genetic determinants that can be indicative of the involvement of common biological mechanisms in both conditions. This second approach is the one we have adopted in this paper.

\section{Materials and Methods}

Here, we determined if candidate genes for mammal domestication are significantly dysregulated in the blood of individuals with ASD. For achieving this, we first compiled an updated list of candidates for domestication by gathering the genes that have been found positively selected in 10 domesticates: Guinea pig, pig, rat, dog, cat, cattle, domesticated fox, horse, rabbit, and sheep [Womack et al., 2005; Trut et al., 2009; Albert et al., 2011; Axelsson et al., 2013; Bellone et al., 2013; Carneiro et al., 2014; Montague et al., 2014; Qanbari et al., 2014; Schubert et al., 2014; Wilkins et al., 2014; Wright et al., 2015; Cagan and Blass, 2016; Freedman et al., 2016; Zapata et al., 2016; Benítez-Burraco et al., 2017; Theofanopoulou et al., 2017; Pendleton et al., 2018]. The list comprised 764 genes (online suppl. list, column A; see www. karger.com/doi/10.1159/000505116). We also considered a list of 89 genes involved in NC development and function, as compiled by Benítez-Burraco et al. [2017] (online suppl. list, column B). These genes include NC markers, genes involved in NC induction and specification or in NC signaling, genes involved in cranial NC differentiation, and candidates for neurochristopathies annotated in the OMIM database (http://omim.org/). Finally, we generated a list of 242 differentially expressed genes (DEGs) in the blood of people with ASD (online suppl. list, column C). For this, we performed microarray analyses of blood samples from 32 patients with ASD (mean age 24.0 years, male:female ratio 50:50) and 30 age-sex-race balanced neurotypical controls (mean age 23.9 years, male:female ratio 50:50). Total RNA was extracted from peripheral blood. All samples had RNA integrity number values $>8$. An Agilent SurePrint G3 Human Gene Expression 8x60K v2 Microarray kit (Agilent Technologies, G4851B) was subsequently used for analyzing transcriptome changes, as described in more detail in Kimura et al. [2019]. Gene expression data can be retrieved from Gene Expression Omnibus (GSE 89594). DEGs were calculated based on diagnosis, age, gender, and RNA integrity number using the Limma R package [Smyth, 2005]. Genes were considered to be differentially expressed when the false discovery rate (FDR) was $<0.1$ and the $\mid$ fold change (FC) $\mid$ was $>1.2$. The Benjamini-Hochberg procedure was used for controlling the FDR in multiple testing [Benjamini and Hochberg, 1995]. We considered protein-coding genes only. We then conducted analyses of gene overlapping between these 3 lists using Fisher exact test $(p<0.05)$.

\section{Results}

We found a significant overlap between DEGs in the blood of subjects with ASD and candidates for mammal domestication. In contrast, we detected no significant overlap between DEGs in subjects with ASD and NC can- 
didates. Table 1 shows the results of Fisher exact tests, as well as the identity of the candidates for domestication that are significantly up- or downregulated in the blood of people with ASD compared to controls (FDR $<0.1$, $|\mathrm{FC}|>1.2)$.

In order to know more about the biological roles played by these genes and about potential connections with the ASD phenotype, we conducted functional enrichment analyses with Enrichr (amp.pharm.mssm.edu/ Enrichr) [Chen et al., 2013; Kuleshov et al., 2016]. We considered biological processes, molecular functions, cellular components, and pathological phenotypes as enriched if $p<0.05$. We found that the protein-coding genes that are dysregulated in the blood of subjects with ASD and that are also candidates for domestication are enriched in processes and functions related to lipid metabolism (like the storage and transport of cholesterol [GO:0010887, GO:0017127], the transport of sterol [GO:0032366], and the homeostasis of phospholipids [GO:0055091]), cell apoptosis (including the apoptosis of muscle cells [GO:0010656] and the endopeptidase activities involved in apoptotic processes [GO:0008635, GO:0097200, and GO:0097153]), the activity of the insulin-like growth factor (GO:0043568, GO:0005159), the physiological roles played by calcineurin (GO:0070886), the regulation of gene expression (via snRNAs [GO:0097322] and transcription co-activators [GO:0001223]), and the posttranslational modifications of proteins, mostly via $\mathrm{NAD}(\mathrm{P})$ (GO:0004029, GO:0016620, GO:0016646) (Fig. 1A, B). Due to the functions in which they are involved, the products of these genes are predicted to be preferentially found in vesicular components of the cell, including the junctional sarcoplasmic reticulum (GO:0014701) and the exocytic vesicles (GO:0070382, GO:0099503) (Fig. 1C). Finally, this set of genes is significantly related to pathological phenotypes entailing skin/hair anomalies, musculoskeletal abnormalities, and hearing impairment (Fig. 1D, E).

\section{Discussion and Conclusion}

In this paper, we have shown that candidate genes for mammal domestication are significantly dysregulated in the blood of individuals with ASD and that this differential expression might account for aspects of the ASD phenotype via the impairment of selected physiological processes. One of these processes is lipid metabolism, which is found altered in ASD [El-Ansary et al., 2019]. Specifically, a significant association exists between hypocholes- terolemia and ASD [Benachenhou et al., 2019] or conditions resulting in autistic features, like Smith-Lemli-Opitz syndrome [Sikora et al., 2006]. Likewise, there is ample evidence of abnormal apoptotic processes in the ASD brain that might account for some of the pathological findings regarding its size, shape, and wiring, and ultimately, for some aspects of its distinctive cognitive and behavioral profile [for discussion, see Wei et al., 2014;

Table 1. Candidate genes for domestication and neural crest development and function that are significantly dysregulated in the blood of subjects with ASD

\begin{tabular}{lrl}
\hline Gene name & $\log$ FC & FDR $p$ value \\
\hline Domestication $(p=0.002)$ & & \\
PAX3 & -0.463 & $1.91 \mathrm{E}-02$ \\
ALDH1L2 & -0.347 & $3.10 \mathrm{E}-02$ \\
LIN28B & -0.315 & $7.76 \mathrm{E}-03$ \\
ALDH16A1 & -0.304 & $6.10 \mathrm{E}-04$ \\
IGF1 & -0.299 & $9.37 \mathrm{E}-02$ \\
ABCG1 & -0.296 & $6.62 \mathrm{E}-02$ \\
CCNT2 & 0.268 & $7.21 \mathrm{E}-02$ \\
IFT81 & 0.270 & $2.99 \mathrm{E}-02$ \\
PTPN4 & 0.299 & $5.35 \mathrm{E}-02$ \\
JPH3 & 0.302 & $1.79 \mathrm{E}-02$ \\
CASP7 & 0.317 & $4.12 \mathrm{E}-02$ \\
UBXN10 & 0.396 & $1.18 \mathrm{E}-02$ \\
TFCP2L1 & 0.441 & $7.40 \mathrm{E}-03$ \\
HOPX & 0.477 & $4.56 \mathrm{E}-02$ \\
\hline Neural crest $(p=0.3126)$ & & \\
PAX3 & -0.463 & $1.91 \mathrm{E}-02$ \\
\hline
\end{tabular}

$\mathrm{FDR}<0.05 ;|\mathrm{FC}|>1.2$.

Fig. 1. Functional enrichment analyses according to Enrichr of the set of genes that are significantly dysregulated in the blood of subjects with ASD and that are also candidates for domestication. A Enrichment in biological processes. B Enrichment in molecular functions. C Enrichment in cellular components. D Enrichment in pathological phenotypes in mammals. E Enrichment in pathological phenotypes in humans. Only the top-10 functions have been included and only if $p<0.05$. The $p$ value was computed using Fisher exact test. Enriched categories are ordered according to their Enrichr Combined Scores. This is a combination of the $p$ value and the $Z$-score calculated by multiplying the 2 scores (Combined Score $=\ln (p$ value $) \times Z$-score $)$. The $Z$-score is computed using a modification to Fisher exact test and assesses the deviation from the expected rank. The Combined Score provides a compromise between both methods, and it is claimed to report the best rankings when compared with the other scoring schemes. For details, see http://amp.pharm.mssm.edu/Enrichr/help\# background \&q=5.

(For figure see next page.) 


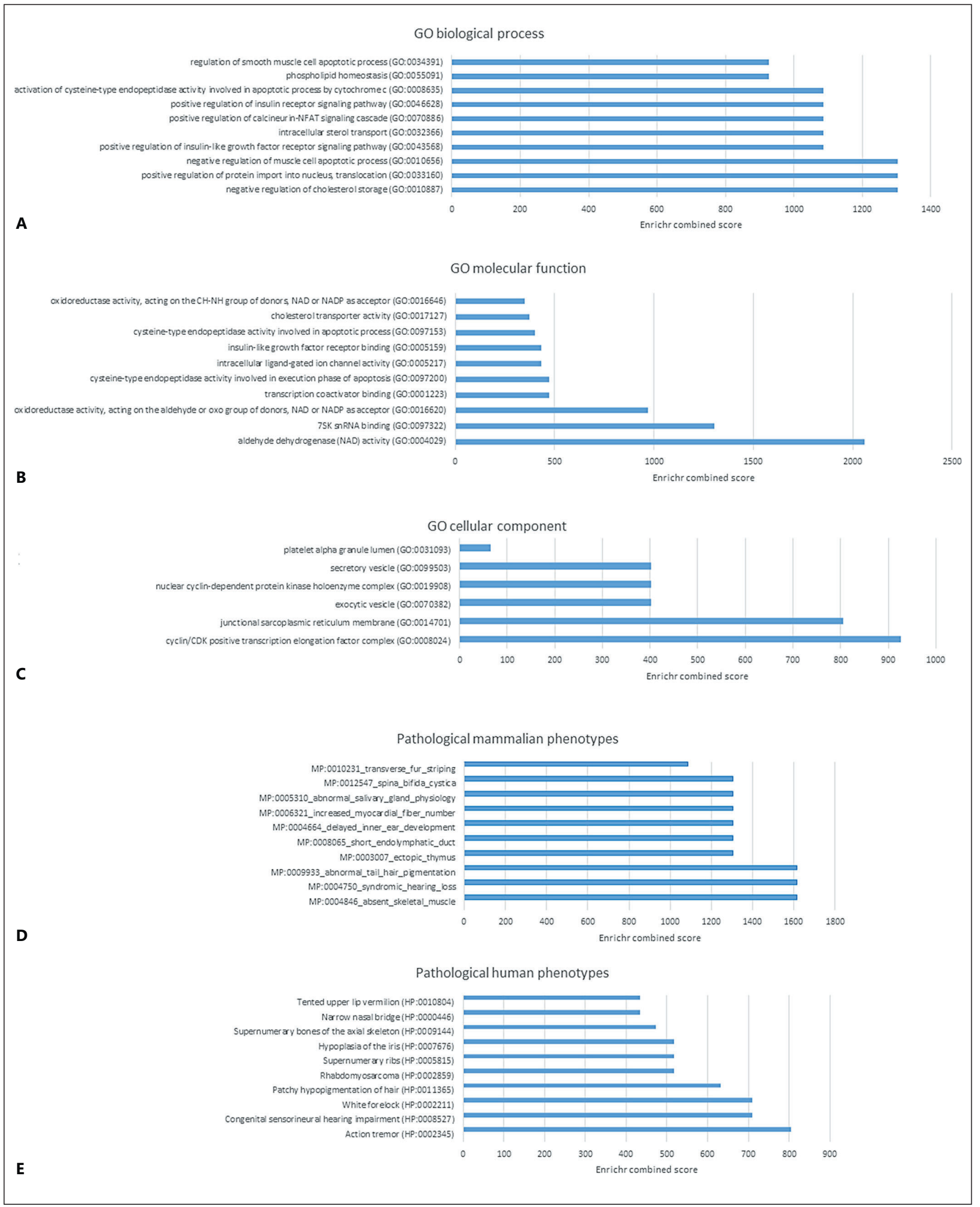


Dong et al., 2018]. Specifically, apoptotic pathways have been found dysregulated in the peripheral blood of autistics [Eftekharian et al., 2019]. Furthermore, one of our DEGs is CASP7, which encodes a caspase and which is repeatedly mentioned as a robust biomarker of metabolic dysfunction in ASD [Khemakhem et al., 2017; El-Ansary et al., 2018]. It is also worth mentioning the enrichment in genes related to the physiological roles of the insulin-like growth factor, including $I G F 1$, which encodes the insulinlike growth factor 1. IGF1 primarily regulates the effects of growth hormone, but it also contributes to neural development, myelinisation, and protection, as well as to synapse formation [for review, see Puche and CastillaCortázar, 2012]. IGF1 levels are abnormally low in individuals with ASD and when administered, IGF1 is expected to ameliorate the symptoms of these conditions [Riikonen, 2016; Steinman, 2019]. Likewise, the enrichment in genes related to calcineurin physiology is worth highlighting, given the association of this phosphatase with intellectual disability in conditions like Down syndrome [Rachidi and Lopes, 2010]. Furthermore, the enrichment in genes involved in gene regulation and/or protein modification should be noted, considering the emergent view of neurodevelopmental disease as resulting from genetic variation in regulatory mechanisms of gene expression [Kubota and Mochizuki, 2016; Geschwind, 2018]. Interestingly enough, autistic features have been found in individuals with mutations in PTPN4, another one of the DEGs in our set and a gene that regulates neuronal development and function, and that is regulated by the product of the main candidate for Rett-syndrome, MECP2 [Williamson et al., 2015; Szczałuba et al., 2018]. Finally, we wish to draw attention to the abnormal phenotypes revealed by the enrichment analysis. The enrichment in musculoskeletal problems can be related to the minor physical anomalies exhibited by subjects with ASD compared to controls, which are more prominent in the craniofacial region, affecting the skull, the ears, and the orofacial area, and which usually correlate with enhanced autistic features [Tripi et al., 2008; Manouilenko et al., 2014; Obafemi-Ajayi et al., 2015; Sacco et al., 2015]. Likewise, regarding the enrichment in genes involved in pigmentation anomalies, it has been observed that hypomelanotic diseases usually co-occur with autistic symptoms, as in hypomelanosis of Ito (OMIM 300337) [Akefeldt and Gillberg, 1991]. The comorbidity between hypomelanosis and ASD has been hypothesized to result from a deficiency in vitamin D [Bakare et al., 2011] to the extent that vitamin D supplementation has been observed to ameliorate symptoms [Jia et al., 2015]. Finally, concerning the enrichment in hearing problems, it should be noted that altered sound processing is commonly observed in people with ASD [Beers et al., 2014; Lucker and Doman, 2015; Do et al., 2017] and that this abnormal sensory processing is hypothesized to impact social functions in these conditions [Thye et al., 2018].

In contrast to the candidate genes for domestication, we found no evidence for differential expression of candidates for NC development and function in the blood of subjects with ASD. In our opinion, this can be due to several reasons. First, the hypothesis that a "domestication syndrome" exists and that this syndrome results from the hypofunction of the $\mathrm{NC}$ is one among several different hypotheses trying to account for the domestication processes in animals [for a critical view, see Sánchez-Villagra et al., 2016]. Actually, most of the NC candidates in our list do not show signals of selection in domesticated animals (there are only 13 overlapping genes). Second, even if the hypofunction of the NC actually triggers domestication, we should expect that dozens of genes downstream the NC inductors are affected and eventually, that some of these genes can be more differentially expressed than most of the NC genes. Third, different cognitive conditions entailing an abnormal presentation of self-domestication features seem to involve different patterns of dysregulation of candidates for domestication and NC development and function. Accordingly, whereas the latter are not differentially expressed in our subjects with ASD, they are differentially dysregulated in people with a different social impairment, namely Williams syndrome [Niego and Benítez-Burraco, 2019]. Finally, human self-domestication seems to be an ongoing process, with different sets of genes having been selected at different moments of our history. Specifically, candidates for domestication and $\mathrm{NC}$ candidates exhibit opposite patterns of enrichment in nonsynonymous single nucleotide polymorphisms when comparing present-day European populations and European samples from 6,000 years ago [Benítez-Burraco et al., 2019]. This could be the case as well with some pathological conditions, like ASD.

Overall, our findings give support to previous claims that one important etiological factor of ASD is the abnormal presentation of the self-domestication phenotype in humans, and ultimately, that a better understanding of this condition will result from the consideration of the evolutionary processes that gave rise to modern human cognition and behavior, human self-domestication being one of them. Certainly, our approach has limitations. First, the size of our sample is reduced. Second, we have analyzed gene expression changes in the blood, whereas most of the relevant changes for the ASD phenotype are expected to 
occur in the brain. Nonetheless, a significant overlapping exists between blood and brain transcriptional profiles, ranging from 20\% [Rollins et al., 2010] to 55\% [Witt et al., 2013], that makes our results reliable. Moreover, there is an increasing interest in finding robust blood biomarkers for ASD that enable an early diagnosis of this condition [for recent reviews, see Bjørklund et al., 2018 or Shen et al., 2019]. Accordingly, we regard the genes we highlight in this paper promising candidates for understanding (and eventually, diagnosing) aspects of the ASD phenotype, whose role in the etiopathogenesis of this condition deserves to be explored in more detail in the next future.

\section{Acknowledgement}

The author thanks Ryo Kimura, Department of Anatomy and Developmental Biology, Graduate School of Medicine of Kyoto University (Japan), for his kind help with data analyses.

\section{Statement of Ethics}

All participants have given their written informed consent, and the study protocol was approved by the ethics committees of each participating institution, as acknowledged in Kimura et al. [2019].

\section{Disclosure Statement}

The author declares no conflict of interests.

\section{Funding Sources}

This research was funded by the Spanish Ministry of Economy and Competitiveness (grant FFI2016-78034-C2-2-P [AEI/FEDER, $\mathrm{UE}]$ ).

\section{References}

Akefeldt A, Gillberg C: Hypomelanosis of Ito in three cases with autism and autistic-like conditions. Dev Med Child Neurol 33:737-743 (1991).

Albert FW, Hodges E, Jensen JD, Besnier F, Xuan $\mathrm{Z}$, et al: Targeted resequencing of a genomic region influencing tameness and aggression reveals multiple signals of positive selection. Heredity 107:205-214 (2011).

-American Psychiatric Association: Diagnostic and Statistical Manual of Mental Disorders (DSM-5), 5th ed (American Psychiatric Association Publishing, Washington 2013).

-Axelsson E, Ratnakumar A, Arendt ML, Maqbool $\mathrm{K}$, Webster MT, et al: The genomic signature of dog domestication reveals adaptation to a starch-rich diet. Nature 495:360-364 (2013).

- Bakare MO, Munir KM, Kinney DK: Association of hypomelanotic skin disorders with autism: links to possible etiologic role of vitamin-D levels in autism? Hypothesis 9:e2 (2011).

- Baxter AJ, Brugha TS, Erskine HE, Scheurer RW, Vos T, Scott JG: The epidemiology and global burden of autism spectrum disorders. Psychol Med 45:601-613 (2015).

- Beers AN, McBoyle M, Kakande E, Dar Santos RC, Kozak FK: Autism and peripheral hearing loss: a systematic review. Int J Pediatr Otorhinolaryngol 78:96-101 (2014).

-Bellone RR, Holl H, Setaluri V, Devi S, Maddodi $\mathrm{N}$, et al: Evidence for a retroviral insertion in TRPM1 as the cause of congenital stationary night blindness and leopard complex spotting in the horse. PLoS One 8:e78280 (2013).

-Benachenhou S, Etcheverry A, Galarneau L, Dubé J, Çaku A: Implication of hypocholesterolemia in autism spectrum disorder and its as- sociated comorbidities: a retrospective case- Do B, Lynch P, Macris EM, Smyth B, Stavrinakis control study. Autism Res 12:1860-1869 (2019).

Benítez-Burraco A, Lattanzi W, Murphy E: Language impairments in ASD resulting from a failed domestication of the human brain. Front Neurosci 10:373 (2016).

Benítez-Burraco A, Di Pietro L, Barba M, Lattanzi W: Schizophrenia and human self-domestication: an evolutionary linguistics approach. Brain Behav Evol 89:162-184 (2017).

Benítez-Burraco A, Chekalin E, Bruskin S, Morozova I: Recent changes in candidate genes for domestication in humans in Europe: focusing on language. https://doi.org/10.1101/684621 (2019).

Benjamini Y, Hochberg Y: Controlling the false discovery rate - a practical and powerful approach to multiple testing. J R Statist Soc B 57: 289-300 (1995).

-Bjørklund G, Meguid NA, El-Ansary A, El-Bana MA, Dadar M, et al: Diagnostic and severitytracking biomarkers for autism spectrum disorder. J Mol Neurosci 66:492-511 (2018).

- Cagan A, Blass T: Identification of genomic variants putatively targeted by selection during dog domestication. BMC Evol Biol 16:10 (2016).

Carneiro M, Rubin C-J, Di Palma F, Albert FW, Alfoldi J, et al: Rabbit genome analysis reveals a polygenic basis for phenotypic change during domestication. Science 345:1074-1079 (2014).

Chen EY, Tan CM, Kou Y, Duan Q, Wang Z, et al: Enrichr: interactive and collaborative HTML5 gene list enrichment analysis tool. BMC Bioinformatics 14:128 (2013). $\mathrm{S}$, et al: Systematic review and meta-analysis of the association of autism spectrum disorder in visually or hearing impaired children. Ophthalmic Physiol Opt 37:212-224 (2017).

Dong D, Zielke HR, Yeh D, Yang P: Cellular stress and apoptosis contribute to the pathogenesis of autism spectrum disorder. Autism Res 11: 1076-1090 (2018).

Eftekharian MM, Komaki A, Oskooie VK, Namvar A, Taheri M, Ghafouri-Fard S: Assessment of apoptosis pathway in peripheral blood of autistic patients. J Mol Neurosci 69: 588-596 (2019).

-El-Ansary A, Bjørklund G, Khemakhem AM, AlAyadhi L, Chirumbolo S, Ben Bacha A: Metabolism-associated markers and childhood autism rating scales (CARS) as a measure of autism severity. J Mol Neurosci 65:265-276 (2018)

-El-Ansary A, Chirumbolo S, Bhat RS, Dadar M, Ibrahim EM, Bjørklund G: The role of lipidomics in autism spectrum disorder. Mol Diagn Ther, Epub ahead of print (2019).

Freedman AH, Schweizer RM, Ortega-Del Vecchyo D, Han E, Davis BW, et al: Demographically-based evaluation of genomic regions under selection in domestic dogs. PLoS Genet 12:e1005851 (2016).

Geschwind DH: Evolving views of human genetic variation and its relationship to neurologic and psychiatric disease. Handb Clin Neurol 147:37-42 (2018).

Geschwind GH, State MW: Gene hunting in autism spectrum disorder: on the path to precision medicine. Lancet Neurol 14:1109-1120 (2015) 
Gyawali S, Patra BN: Autism spectrum disorder: trends in research exploring etiopathogenesis. Psychiatry Clin Neurosci 73:466-475 (2019).

Hare B: Survival of the friendliest: Homo sapiens evolved via selection for prosociality. Annu Rev Psychol 68:155-186 (2017).

- Jia F, Wang B, Shan L, Xu Z, Staal WG, Du L: Core symptoms of autism improved after vitamin D supplementation. Pediatrics 135:e196-198 (2015).

Khemakhem AM, Frye RE, El-Ansary A, Al-Ayadhi L, Bacha AB: Novel biomarkers of metabolic dysfunction is autism spectrum disorder: potential for biological diagnostic markers. Metab Brain Dis 32:1983-1997 (2017).

- Kimura R, Swarup V, Tomiwa K, Gandal MJ, Parikshak NN, et al: Integrative network analysis reveals biological pathways associated with Williams syndrome. J Child Psychol Psychiatry 60:585-598 (2019).

Kubota T, Mochizuki K: Epigenetic effect of environmental factors on autism spectrum disorders. Int J Environ Res Public Health 13:E504 (2016).

Kuleshov MV, Jones MR, Rouillard AD, Fernandez NF, Duan Q, et al: Enrichr: a comprehensive gene set enrichment analysis web server 2016 update. Nucleic Acids Res 44(W1):W90-97 (2016).

Kumar S, Reynolds K, Ji Y, Gu R, Rai S, Zhou CJ: Impaired neurodevelopmental pathways in autism spectrum disorder: a review of signaling mechanisms and crosstalk. J Neurodev Disord 11:10 (2019).

-Lucker JR, Doman A: Neural mechanisms involved in hypersensitive hearing: helping children with ASD who are overly sensitive to sounds. Autism Res Treat 2015:369035 (2015).

-Manouilenko I, Eriksson JM, Humble MB, Bejerot S: Minor physical anomalies in adults with autism spectrum disorder and healthy controls. Autism Res Treat 2014:743482 (2014).

-Montague MJ, Li G, Gandolfi B, Khan R, Aken BL, et al: Comparative analysis of the domestic cat genome reveals genetic signatures underlying feline biology and domestication. Proc Natl Acad Sci USA 111:17230-17235 (2014).

Niego A, Benítez-Burraco A: Williams syndrome, human self-domestication, and language evolution. Front Psychol 10:521 (2019).

-Obafemi-Ajayi T, Miles JH, Takahashi TN, Qi W, Aldridge K, et al: Facial structure analysis separates autism spectrum disorders into meaningful clinical subgroups. J Autism Dev Disord 45:1302-1317 (2015).
Pendleton AL, Shen F, Taravella AM, Emery S, Veeramah KR, et al: Comparison of village dog and wolf genomes highlights the role of the neural crest in dog domestication. BMC Biol 16:64 (2018).

Puche JE, Castilla-Cortázar I: Human conditions of insulin-like growth factor-I (IGF-I) deficiency. J Transl Med 10:224 (2012).

Qanbari S, Pausch H, Jansen S, Somel M, Strom TM, et al: Classic selective sweeps revealed by massive sequencing in cattle. PLoS Genet 10:e1004148 (2014).

Rachidi M, Lopes C: Molecular and cellular mechanisms elucidating neurocognitive basis of functional impairments associated with intellectual disability in Down syndrome. Am J Intellect Dev Disabil 115:83-112 (2010).

Riikonen R: Treatment of autistic spectrum disorder with insulin-like growth factors. Eur J Paediatr Neurol 20:816-823 (2016).

Rollins B, Martin MV, Morgan L, Vawter MP: Analysis of whole genome biomarker expression in blood and brain. Am J Med Genet B Neuropsychiatr Genet 153B:919-936 (2010).

-Sacco R, Gabriele S, Persico AM: Head circumference and brain size in autism spectrum disorder: a systematic review and meta-analysis. Psychiatry Res 234:239-251 (2015).

Sánchez-Villagra MR, Geiger M, Schneider RA: The taming of the neural crest: a developmental perspective on the origins of morphological covariation in domesticated mammals. $\mathrm{R}$ Soc Open Sci 3:160107 (2016).

- Schubert M, Jónsson H, Chang D, Der Sarkissian C, Ermini L, et al: Prehistoric genomes reveal the genetic foundation and cost of horse domestication. Proc Natl Acad Sci USA 111:E5661-5669 (2014).

-Shen L, Zhao Y, Zhang H, Feng C, Gao Y, et al: Advances in biomarker studies in autism spectrum disorders. Adv Exp Med Biol 1118: 207-233 (2019).

Sikora DM, Pettit-Kekel K, Penfield J, Merkens, LS, Steiner RD: The near universal presence of autism spectrum disorders in children with Smith-Lemli-Opitz syndrome. Am J Med Genet A 140:1511-1518 (2006).

Smyth GK: Limma: linear models for microarray data, in Gentleman R, Carey V, Huber W, Irizarry R, Dudoit S (eds): Bioinformatics and Computational Biology Solutions using R and Bioconductor, pp 397-420 (Springer, New York 2005).

Steinman G: IGF - autism prevention/amelioration. Med Hypotheses 122:45-47 (2019).

Sullivan JM, De Rubeis S, Schaefer A: Convergence of spectrums: neuronal gene network states in autism spectrum disorder. Curr Opin Neurobiol 59:102-111 (2019).
Szczałuba K, Chmielewska JJ, Sokolowska O, Rydzanicz M, Szymańska K, et al: Neurodevelopmental phenotype caused by a de novo PTPN4 single nucleotide variant disrupting protein localization in neuronal dendritic spines. Clin Genet 94:581-585 (2018).

Theofanopoulou C, Gastaldon S, O'Rourke T, Samuels BD, Martins PT, et al: Self-domestication in Homo sapiens: insights from comparative genomics. PLoS One 12:e185306 (2017).

Thomas J, Kirby S: Self domestication and the evolution of language. Biol Philos 33:9 (2018).

Thye MD, Bednarz HM, Herringshaw AJ, Sartin EB, Kana RK: The impact of atypical sensory processing on social impairments in autism spectrum disorder. Dev Cogn Neurosci 29: 151-167 (2018).

Toro R, Konyukh M, Delorme R, Leblond C, Chaste $\mathrm{P}$, et al: Key role for gene dosage and synaptic homeostasis in autism spectrum disorders. Trends Genet 26:363-372 (2010).

Tripi G, Roux S, Canziani T, Bonnet Brilhault F, Barthélémy C, Canziani F: Minor physical anomalies in children with autism spectrum disorder. Early Hum Dev 84:217-223 (2008).

Trut L, Oskina I, Kharlamova A: Animal evolution during domestication: the domesticated fox as a model. Bioessays 31:349-360 (2009).

Wei H, Alberts I, Li X: The apoptotic perspective of autism. Int J Dev Neurosci 36:13-18 (2014).

Wilkins AS, Wrangham RW, Fitch WT: The "domestication syndrome" in mammals: a unified explanation based on neural crest cell behavior and genetics. Genetics 197:795-808 (2014).

Williamson SL, Ellaway CJ, Peters GB, Pelka GJ, Tam PP, Christodoulou J: Deletion of protein tyrosine phosphatase, non-receptor type 4 (PTPN4) in twins with a Rett syndrome-like phenotype. Eur J Hum Genet 23:1171-1175 (2015).

Witt SH, Sommer WH, Hansson AC, Sticht C, Rietschel M, Witt CC: Comparison of gene expression profiles in the blood, hippocampus and prefrontal cortex of rats. In Silico Pharmacol 1:15 (2013).

Womack JE: Advances in livestock genomics: opening the barn door. Genome Res 15:16991705 (2005).

Wright D: The genetic architecture of domestication in animals. Bioinform Biol Insights 9(Suppl 4):11-20 (2015).

Zapata I, Serpell JA, Álvarez CE: Genetic mapping of canine fear and aggression. BMC Genomics 17:572 (2016). 